\title{
An Embedded-Atom-Method Model for Alkali-Metal Vibrations
}

\author{
R B Wilson and D M Riffe \\ Physics Department, Utah State University, Logan, UT 84322-4415
}

\begin{abstract}
We present an embedded-atom-method (EAM) model that accurately describes vibrational dynamics in the alkali metals $\mathrm{Li}, \mathrm{Na}, \mathrm{K}, \mathrm{Rb}$, and Cs. Bulk dispersion curves, frequency-moment Debye temperatures, and temperature-dependent entropy Debye temperatures are all in excellent agreement with experimental results. The model is also well suited for studying surface vibrational dynamics in these materials, as illustrated by calculations for the $\mathrm{Na}(110)$ surface.
\end{abstract}

PACS numbers: 63.20.dh, 68.35.Ja

\section{Introduction}

The embedded atom method (EAM), originally developed by Daw and Baskes to study fcc metals $[1,2]$, is a semi-empirical, many-body approach that can be used to calculate a wide variety of materials properties such as lattice stability, thermal expansion, surface and interface structure, defect properties, and vibrational dynamics [3]. EAM models have had particular success at predicting both bulk and surface phonons in fcc metals such as $\mathrm{Cu}$ and $\mathrm{Ag}[3,4,5,6,7]$.

In contrast, many EAM models do a rather poor job of predicting the vibrational properties of bcc metals, including the simple metals $\mathrm{Li}, \mathrm{Na}, \mathrm{K}, \mathrm{Rb}$, and $\mathrm{Cs}$. This can partly be ascribed to the longer range and/or angular nature of the forces in the less closed packed bcc lattice, which makes appropriate EAM modeling more complex than for fcc materials. The lack of success can also be ascribed, in some cases, to the use of less-than-ideal input parameters in constructing the EAM potentials. There are, however, a few encouraging counterexamples to this generally poor performance, notably the EAM potentials of Chantasiriwan and Milstein (CM) for Li and K, which do a relatively good job at predicting phonon dispersion for these two metals [8]. The CM results suggest that the EAM has the potential to accurately describe the vibrational properties of the alkali metals and also, perhaps, other bcc metals.

Here we present a normalized EAM model, based on the pair-potential formulation of Wang and Boercker (WB) [9] and analytic embedding energy of Johnson and Oh (JO) [10], that accurately predicts bulk vibrational properties associated with all of the alkali metals. These properties include not only dispersion curves, but also frequencymoment and vibrational-entropy Debye temperatures. The quantitative agreement that 
we obtain with experiment is superior to that obtained from other EAM treatments of alkali-metal lattice dynamics. We also apply the model to the most studied allkalimetal surface, $\mathrm{Na}(110)$. We identify surface localized modes and calculate directionally resolved Debye temperatures for near-surface planes of atoms.

\section{EAM model}

The EAM describes the total potential energy associated with the atomic positions as a sum of pair potentials $\phi\left(r_{i j}\right)$ and embedding energies $F\left(\rho_{i}\right)$

$$
U=\frac{1}{2} \sum_{i j} \phi\left(r_{i j}\right)+\sum_{i} F\left(\rho_{i}\right)
$$

where $i$ and $j(i \neq j)$ label the atoms in the solid, $r_{i j}$ is the distance between atoms $i$ and $j$, and $\rho_{i}$ is the electron density at the position of atom $i$ due to all of the other atoms in the solid. It is assumed that this density can be written as a sum of individual atomic densities $f\left(r_{i j}\right)$

$$
\rho_{i}=\sum_{j} f\left(r_{i j}\right)
$$

Specifying the functions $\phi(r), F(\rho)$, and $f(r)$ establishes an EAM model for a particular material.

To describe the alkali metals we use previously introduced forms for these three functions. Our choices for these functions are predicated on the ability of the resulting models to (i) accurately describe bulk-phonon spectra and (ii) produce realistic values for surface relaxation. Both abilities are necessary for accurately predicting surface phonons. After considering a number of potential functions we settled on the pairpotential formulation of WB [9],

$$
\phi(r)=\sum_{n=0}^{6} K_{n}\left(\frac{r}{r_{1}}-1\right)^{n} \exp \left(-n \alpha\left(\frac{r}{r_{1}}-1\right)^{2}\right),
$$

and the embedding-energy functions of JO [10, 11],

$$
F(\rho)=-\left(E_{c o h}-E_{1 v}^{U F}\right)\left[1-\lambda \ln \left(\frac{\rho}{\rho_{e}}\right)\right]\left(\frac{\rho}{\rho_{e}}\right)^{\lambda}
$$

and

$$
f(r)=f_{1} \exp \left(-\beta\left(\frac{r}{r_{1}}-1\right)\right)
$$

In $(3)-(5)$ the parameter $r_{1}$ is the nearest-neighbor distance, $E_{c o h}$ the cohesive energy, and $E_{1 v}^{U F}$ the (unrelaxed) vacancy formation energy; all are obtained from experimental measurements. The parameters $K_{n}$ in (3) are also determined from experimental inputs, as described below. The exact value of the nearest-neighbor density $f_{1}$ in (4) does not impact the model because the embedding function $F(\rho)$ only depends upon the ratio 
of the charge density $\rho$ to its equilibrium value $\rho_{e}$; for simplicity we set its value to 1 . Following JO we choose $\beta$ to have the value of 6 [10]. Because $F(\rho)$ is a minimum at the equilibrium density $\rho_{e}$, this model is known as a normalized EAM model.

Following WB [9], we determine the seven pair-potential parameters $K_{n}$ in (3) using

$$
\begin{aligned}
& 0=4 r_{1} \phi_{1}^{\prime}+3 r_{2} \phi_{2}^{\prime}+6 r_{3} \phi_{3}^{\prime}+12 r_{4} \phi_{4}^{\prime}+4 r_{5} \phi_{5}^{\prime}, \\
& 15 \Omega G=4 r_{1}^{2}\left(\phi_{1}^{\prime \prime}+\phi_{2}^{\prime \prime}+4 \phi_{3}^{\prime \prime}+11 \phi_{4}^{\prime \prime}+4 \phi_{5}^{\prime \prime}\right), \\
& 3 \Omega C^{\prime}=2 r_{1}^{2}\left(\phi_{2}^{\prime \prime}-\frac{\phi_{2}^{\prime}}{r_{2}}+\phi_{3}^{\prime \prime}-\frac{\phi_{3}^{\prime}}{r_{3}}+\frac{64}{11} \phi_{4}^{\prime \prime}-\frac{64}{11} \frac{\phi_{4}^{\prime}}{r_{4}}\right), \\
& E_{1 v}^{U F}=-4 \phi_{1}-3 \phi_{2}-6 \phi_{3}-12 \phi_{4}-4 \phi_{5}, \\
& \frac{3}{16} M \omega_{100}^{2}=\phi_{1}^{\prime \prime}+2 \frac{\phi_{1}^{\prime}}{r_{1}}+3 \phi_{4}^{\prime \prime}+6 \frac{\phi_{4}^{\prime}}{r_{4}}, \\
& \frac{1}{4} M \omega_{\frac{1}{2} \frac{1}{2} 0}^{2}=\frac{4}{3} \phi_{1}^{\prime \prime}+\frac{2}{3} \frac{\phi_{1}^{\prime}}{r_{1}}+\phi_{2}^{\prime \prime}+\frac{\phi_{2}^{\prime}}{r_{2}}+\phi_{3}^{\prime \prime}+3 \frac{\phi_{3}^{\prime}}{r_{3}}+\frac{12}{11} \phi_{4}^{\prime \prime}+\frac{54}{11} \frac{\phi_{4}^{\prime}}{r_{4}},
\end{aligned}
$$

and

$$
\frac{3}{8} M \omega_{\frac{1}{2} \frac{1}{2} \frac{1}{2}}^{2}=\phi_{1}^{\prime \prime}+2 \frac{\phi_{1}^{\prime}}{r_{1}}+\frac{3}{2} \phi_{2}^{\prime \prime}+3 \frac{\phi_{2}^{\prime}}{r_{2}}+3 \phi_{4}^{\prime \prime}+6 \frac{\phi_{4}^{\prime}}{r_{4}}+2 \phi_{5}^{\prime \prime}+4 \frac{\phi_{5}^{\prime}}{r_{5}} .
$$

In these equations $\phi_{i}^{\prime}=d \phi / d r$ and $\phi_{i}^{\prime \prime}=d^{2} \phi / d r^{2}$ evaluated at $r_{i}$, where $r_{i}$ is the equilibrium distance to the $i$ th shell of neighbors. In addition to the vacancy formation energy $E_{1 v}^{U F}$, experimental inputs to these equations are the atomic mass $M$, atomic volume $\Omega=a_{0}^{3} / 2\left(a_{0}=\right.$ lattice constant), elastic constants $G=\left(C_{11}-C_{12}+3 C_{44}\right) / 5$ and $C^{\prime}=\left(C_{11}-C_{12}\right) / 2$, and three longitudinal-mode (angular) frequencies $\omega_{i j k}$, where the subscript denotes the location of the mode at the boundary of the Brillouin zone (BZ). As is evident in (6) - (12) the pair potential for neighbors beyond the fifth shell is assumed to be zero. A simple scheme can be used to smoothly cut off the potential, as expressed by (3), between the fifth and sixth neighbor distances [12]. Similarly, $f(r)$ is assumed to smoothly go to zero for $r$ between $r_{5}$ and $r_{6}$.

We determine the best values for the exponents $\lambda$ and $\alpha$ by comparing calculated and experimental dispersion curves (discussed in detail in section 3). Surface relaxation (see section 4.1) is also considered in choosing $\lambda$. This approach differs from that of JO, who use a universal value, $\lambda=\sqrt{1 / 8}(=0.354)$, based on a comparison of their model with early first-principles calculations of the atomic potentials [10]. This is fairly close to our values for $\mathrm{Li}$ and $\mathrm{Na}$. However, we find that $\lambda=0.354$ results in [100] longitudinal frequencies for $\mathrm{K}, \mathrm{Rb}$, and $\mathrm{Cs}$ that are significantly smaller than the experimental measurements. We also note that our values of $\alpha$ for all of the alkali metals (which are all close to 0.5 ) contrast with the choice made by WB, who set $\alpha=0$ for their modeling of $\mathrm{Li}, \mathrm{Na}$, and $\mathrm{K}[9]$.

Table 1 lists values of the experimental parameters that we have used to construct the EAM potentials for the alkali metals. We emphasize that accurate values of the experimentally determined parameters are crucial in order to successfully predict 
Table 1. Experimental and adjustable $(\alpha$ and $\lambda)$ parameters used to construct the EAM potentials for the alkali metals.

\begin{tabular}{|c|c|c|c|c|c|}
\hline & $\mathrm{Li}$ & $\mathrm{Na}$ & $\mathrm{K}$ & $\mathrm{Rb}$ & Cs \\
\hline$M(\mathrm{amu})$ & 6.94 & 23.0 & 39.1 & 85.5 & 132.9 \\
\hline$E_{c o h}(\mathrm{eV})^{\mathrm{a}}$ & 1.63 & 1.11 & 0.93 & 0.85 & 0.80 \\
\hline$E_{1 v}^{U F}(\mathrm{eV})^{\mathrm{b}}$ & 0.40 & 0.36 & 0.35 & 0.30 & 0.28 \\
\hline$T(\mathrm{~K})$ & 98 & 90 & 9 & 12 & 50 \\
\hline$a_{0}(\mathrm{~nm})^{\mathrm{c}}$ & 0.348 & 0.424 & 0.524 & 0.559 & 0.605 \\
\hline$G(100 \mathrm{Mbar})^{\mathrm{d}}$ & 6.90 & 3.80 & 1.86 & 1.42 & 1.00 \\
\hline$C^{\prime}(100 \mathrm{Mbar})^{\mathrm{d}}$ & 1.10 & 0.72 & 0.37 & 0.27 & 0.21 \\
\hline$\nu_{100}(\mathrm{Thz})^{\mathrm{e}}$ & 9.00 & 3.58 & 2.21 & 1.38 & 0.96 \\
\hline$\nu_{\frac{1}{2} \frac{1}{2} \frac{1}{2}}(\mathrm{Thz})^{\mathrm{e}}$ & 7.00 & 2.88 & 1.78 & 1.13 & 0.78 \\
\hline$\nu_{\frac{1}{2} \frac{1}{2} 0}(\mathrm{Thz})^{\mathrm{e}}$ & 9.10 & 3.82 & 2.40 & 1.50 & 1.07 \\
\hline$\alpha$ & 0.505 & 0.514 & 0.470 & 0.495 & 0.450 \\
\hline$\lambda$ & 0.30 & 0.38 & 0.48 & 0.45 & 0.48 \\
\hline
\end{tabular}

a Cohesive energies from [13].

b Vacancy formation energies from [14] (Li), [15] (Na), [16] (K), [17] (Rb, Cs).

${ }^{c}$ Lattice constants from [18] (Li), [19, 20] (Na), [21] (K), [20] (Rb), [18] (Cs).

${ }^{d}$ Elastic constants from [22] (Li), [23, 24] (Na), [25] (K), [26] (Rb), [27] (Cs).

e Vibrational frequencies from [28] (Li), [29] (Na), [30] (K), [31] (Rb), [32] (Cs).

vibrational properties, and so we have carefully perused the literature to obtain what we judge to be the most accurate values. The temperature listed for each metal is the lowest temperature where neutron scattering has been used to obtain dispersion curves for that metal. The values for the lattice constants $a_{0}$ and elastic constants $G$ and $C^{\prime}$ are appropriate for the listed temperatures. The frequencies $\nu_{i j k}=\omega_{i j k} /(2 \pi)$ are obtained from the experimental dispersion curves shown in figure 1. Table 1 also lists best values for the two adjustable parameters $\alpha$ and $\lambda$. Table 2 lists the values of the pair-potential parameters $K_{n}$, which are determined using (6) - (12) with the input parameters displayed in table 1. (As an aside, we note that in addition to the following discussion of vibrational properties, in Appendix A we briefly discuss the relative stability of bcc, fcc, and hcp lattices as calculated with our EAM potentials.)

\section{Bulk dynamics}

\subsection{Dispersion curves}

Figure 1 displays bulk phonon-dispersion curves for all of the alkali metals. Our calculations (solid lines) are displayed along with experimental results obtained using neutron scattering (filled circles). For comparison, results from two other models are also displayed in the figure: the dashed lines are calculated using the original bcc EAM model of JO [10], and the dotted lines are calculated using the EAM potentials of CM [8].

As is evident in the figure, our calculated dispersion curves are in excellent 
Table 2. Pair-potential parameters $K_{n}$ for the alkali metals.

\begin{tabular}{lrrrrr}
\hline & \multicolumn{1}{c}{$\mathrm{Li}$} & \multicolumn{1}{c}{$\mathrm{Na}$} & \multicolumn{1}{c}{$\mathrm{K}$} & \multicolumn{1}{c}{$\mathrm{Rb}$} & \multicolumn{1}{c}{$\mathrm{Cs}$} \\
\hline$K_{0}$ & -0.06480 & -0.04760 & -0.04455 & -0.04589 & -0.03328 \\
$K_{1}$ & -0.02748 & -0.06872 & -0.11352 & -0.10437 & -0.13750 \\
$K_{2}$ & 1.93532 & 1.58403 & 1.60760 & 1.58272 & 1.45194 \\
$K_{3}$ & -12.89694 & -6.00685 & -5.29804 & -5.94188 & -4.64698 \\
$K_{4}$ & 48.45565 & 15.59265 & 11.08178 & 15.63272 & 9.92710 \\
$K_{5}$ & -84.11401 & -25.44532 & -14.96996 & -24.48760 & -13.75393 \\
$K_{6}$ & 52.07501 & 16.50906 & 8.64077 & 15.00282 & 8.07422 \\
\hline
\end{tabular}

agreement with the experimental results, with two slight systematic deviations: (i) the higher frequency transverse mode along [ $q q 0]$ is overestimated near the zone edge, and (ii) the longitudinal frequencies near $[q q q]=[0.75,0.75,0.75]$ are also overestimated. As discussed in detail below, this excellent agreement can be attributed to the use of (i) accurate elastic constants and (ii) bulk-phonon frequencies in constructing the pair potentials.

The JO model [10], which only considers interactions between nearest neighbors $(\mathrm{NN})$ and next nearest neighbors (NNN) is the precursor to the model that we use here. The JO model's inputs for the pair potential are simply $a_{0}, E_{c o h}, G$, and $C^{\prime}$. For the curves displayed in figure 1 we have used the same values for these parameters (and $\lambda$ ) as for the curves calculated with our model. Thus, there is good agreement between our model and the JO model near the zone center, where the elastic constants and $\lambda$ largely determine the frequencies. Near the BZ zone edge, however, the JO model systematically underestimates the frequencies of most of the modes. This discrepancy illustrates the advantage of the WB pair potential, in that it also uses three phonon frequencies as inputs. With regards to the JO model, we note that Guellil and Adams (GA) previously published dispersion curves using this model [12]; however, owing to their use of room-temperature (RT) elastic constants (rather than values appropriate for the temperatures associated with the experimental dispersion curves), all frequencies calculated by GA are systematically lower than the experimental frequencies [12].

EAM potentials for $\mathrm{Li}, \mathrm{Na}, \mathrm{K}$, and $\mathrm{Rb}$ have been presented by $\mathrm{CM}$ in conjunction with their model, which uses not only second-order elastic constants $\left(C_{i j}\right)$ but also third-order elastic constants $\left(C_{i j k}\right)$ to construct the model [8]. As figure 1 shows, the $\mathrm{CM}$ potentials for $\mathrm{Li}, \mathrm{Na}$, and $\mathrm{K}$ produce dispersion curves that are overall somewhat better that the JO curves, but still inferior to the results from our model. For Rb it is curious that the CM model underestimates all frequencies. As with the curves calculated by GA using the JO model, this appears to be the result of using inaccurate second-order elastic constants in constructing the Rb potential.

We note that several other EAM models have been used to calculate alkali-metal dispersion curves $[33,34,35]$. All of these are modified analytic EAM (MAEAM) models, which have an additional potential-energy term that depends upon the square of the 

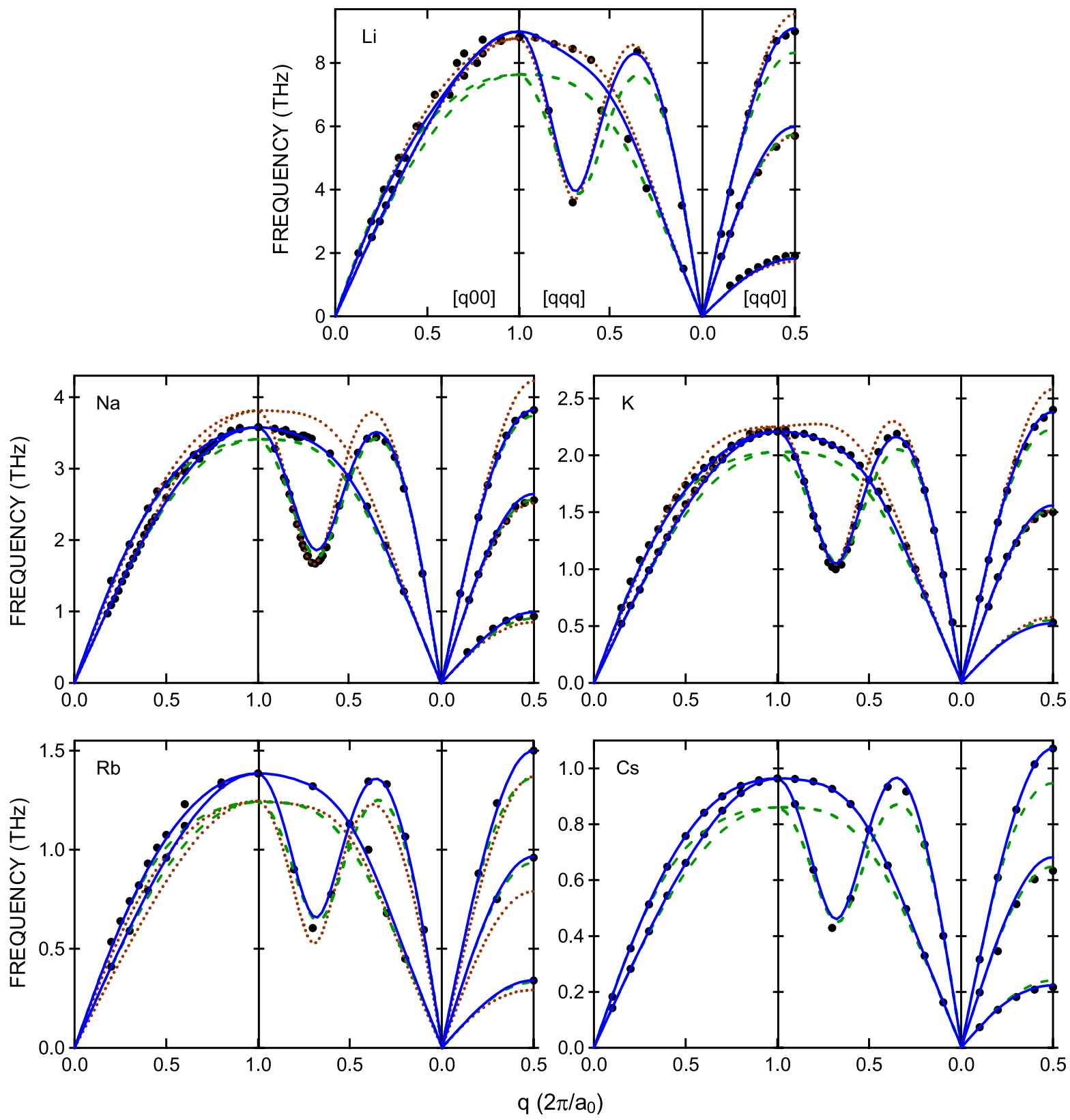

Figure 1. Bulk alkali-metal phonon-dispersion curves. Filled circles are experimental data, [28] (Li), [29] (Na), [30] (K), [31] (Rb), [32] (Cs). Solid, dashed, and dotted lines are present, JO, and CM EAM model calculations, respectively. See text for details.

electron density. The MAEAM model discussed by $\mathrm{Hu}$ and Masahiro (HM) produces results similar to the those of the CM model [33]. Zhang and coworkers have published two sets of dispersion curves based on the MAEAM approach; overall, the degree of agreement with experiment is comparable to that of the JO-model calculated curves displayed in figure $1[34,35]$. 


\subsection{Debye temperatures}

Being able to match experimental frequencies along high-symmetry directions is certainly a prerequisite for accurate modeling of vibrational dynamics. However, such accuracy does not necessarily guarantee likewise success throughout the BZ. In order to assess our model's accuracy at describing all vibrational modes, we calculate the bulk vibrational density of states $g(\omega)$ and then use $g(\omega)$ to compute frequency-moment $(n)$ Debye temperatures [36]

$$
\Theta_{D}(n)=\frac{\hbar}{k_{B}}\left(\frac{n+3}{3} \frac{\int \omega^{n} g(\omega) d \omega}{\int g(\omega) d \omega}\right)^{1 / n} \quad(n>-3, n \neq 0)
$$

and

$$
\Theta_{D}(0)=\frac{\hbar}{k_{B}} \exp \left(\frac{1}{3}+\frac{\int \ln (\omega) g(\omega) d \omega}{\int g(\omega) d \omega}\right) . \quad(n=0)
$$

In addition to these Debye temperatures the $n=-3$ Debye temperature is defined as the Debye temperature that describes the low-temperature limit of the vibrational specific heat. For a Debye-model description of vibrations (where $g(\omega) \sim \omega^{2}$ ) all frequencymoment $\Theta_{D}(n)$ 's are identical; for a real solid the variations in $\Theta_{D}(n)$ with $n$ provide insight into variations of the actual density of states from that of the simple Debye model. Importantly, certain moments govern specific physical properties: in addition to the low-temperature specific heat being governed by $n=-3$, the high-temperature meansquared amplitude is governed by $n=-2$, the low-temperature mean-squared amplitude by $n=-1$, the high-temperature entropy by $n=0$, and the high-temperature specific heat by $n=2$ [36]. This means, for example, that the Debye temperature deduced from the temperature dependence of x-ray diffraction-peak intensity is equal to $\Theta_{D}(-2)$ when the measurements are made near room temperature. As will be seen below, this Debye temperature may be substantially different from a Debye temperature deduced with with another experimental probe.

In figure 2 we compare our calculated values (squares) of $\Theta_{D}(n)$ with experimental values (circles with error bars) extracted from temperature-dependent specific-heat measurements [17]. Values calculated with the JO (triangles) and CM (diamonds) models are also displayed. Our calculated values for $\mathrm{Na}, \mathrm{K}$, and $\mathrm{Rb}$ all lie within the experimental error bars. For Cs the agreement is also excellent at small values of $n$; at larger values of $n$ the calculated values are slightly low, but still within $\sim 5 \%$ of the experimental values. The disagreement for $\mathrm{Li}$ at small values of $n$ is due to the 80 K martensitic bcc to closed-packed phase transition [37]. While Na undergoes a similar transition, it is possible to determine the low-temperature thermodynamic properties of bcc Na [17]. As expected from the comparisons of dispersion curves in figure 1, the JO model does reasonably well for the smallest values of $n$ but becomes progressively worse as $n$ increases. Results using the CM model are also commensurate with the dispersion-curve comparisons, particularly the too-high values for larger $n$ values for $\mathrm{K}$ and $\mathrm{Na}$ and the overall low values for $\mathrm{Rb}$. 

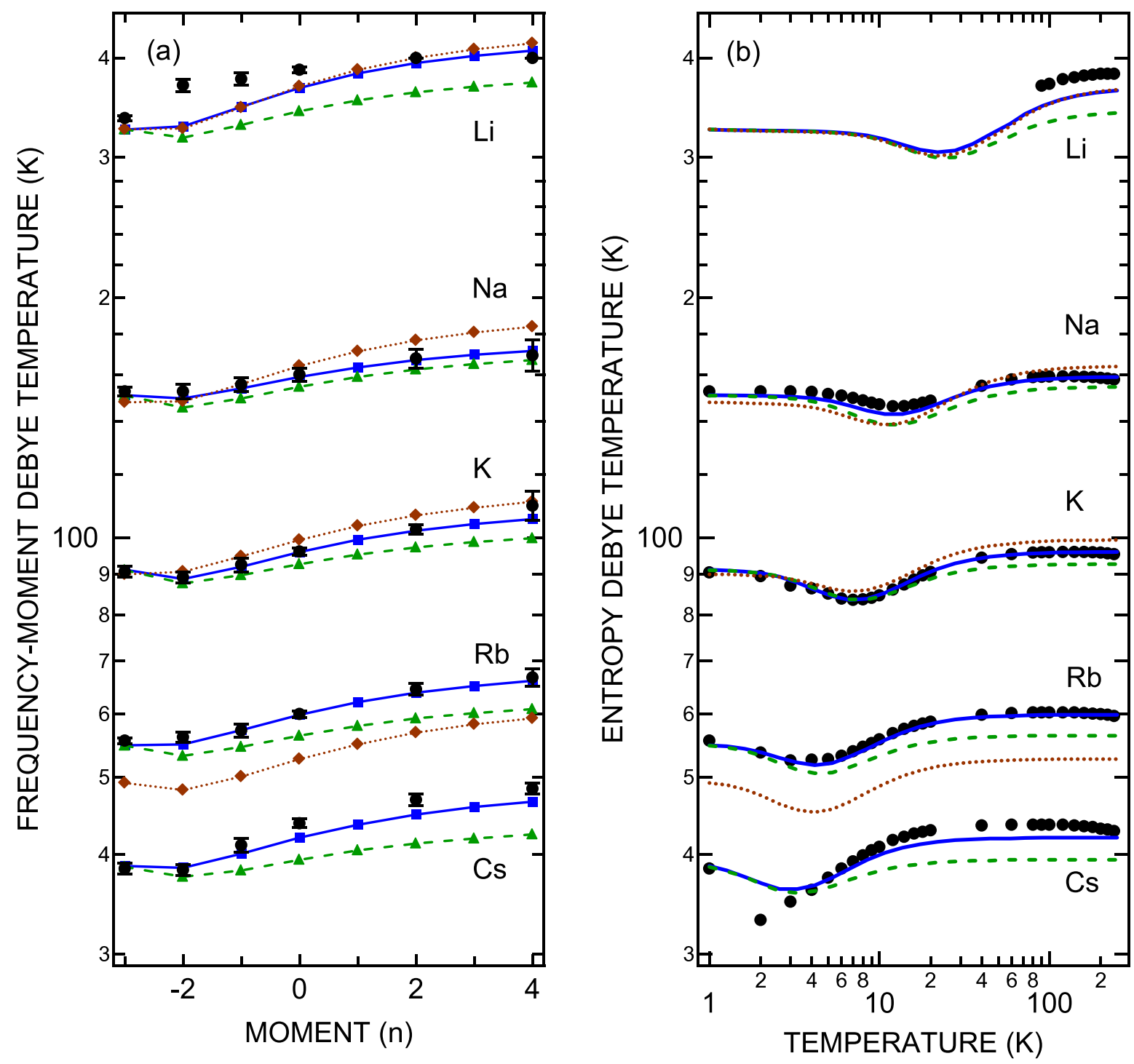

Figure 2. Bulk Debye temperatures for the alkali metals. (a) Frequency-moment Debye temperatures $\Theta_{D}(n)$. Circles with error bars are experimental results; squares with solid lines, present EAM models; triangles with dashed lines, JO EAM models; diamonds with dotted lines, CM EAM models. (b) Temperature-dependent entropy Debye temperatures $\Theta_{D}^{S}(T)$. Circles are experimental results; solid lines, present EAM models; dashed lines, JO EAM models; dotted lines, CM EAM models.

Also plotted in figure 2 are values for the temperature-dependent entropy Debye temperatures $\Theta_{D}^{S}(T)$; the experimental results are again obtained from temperaturedependent specific-heat measurements [17]. Similar to the results for the dispersion curves and frequency-moment Debye temperatures, the predictions from our EAM potentials are generally superior to those from the JO and CM EAM models. 


\section{Surface dynamics}

\subsection{Surface relaxation}

Because the localization of vibrational modes at a surface can be quite sensitive to structure, it is desirable that any theory used to calculate surface vibrations be able to accurately predict surface relaxation. As shown in table 3 our EAM potentials predict relaxations for low-index $\mathrm{Li}$ and $\mathrm{Na}$ surfaces that are quite similar to those predicted by density-functional theory (DFT): predicted relaxations are all inward (or zero) and at most only a few percent of the inter-layer spacing.

It is instructive to compare our computed relaxations with those predicted by two other EAM models, also listed in table 3. The values calculated by GA with the JO model are almost identical to ours [12]. In contrast, the first-layer relaxations calculated by Sklyadneva, Bertsch, and Chulkov (SBC) [38, 39], using an EAM model that is based on the original Daw and Baskes fcc model, are all positive (expansion), in disagreement with all other calculations shown in table 3. From these comparisons we can infer the following. First, the fact that the JO model uses a different pair potential but the same embedding-energy description that we do indicates that the embedding-energy formulation is key to correctly predicting accurate relaxations. This is not surprising because without the embedding-energy contribution the (typically) repulsive pair interaction between nearest neighbors usually produces an expansion between the first and second surface layers. Thus, the fact that the SBC model predicts positive expansions for all four surfaces suggests that their embedding energy does not vary strongly enough with lattice constant, allowing the pair-potential interaction to dominate near-surface relaxation.

\section{2. $\mathrm{Na}(110)$ vibrations}

To investigate vibrational dynamics at alkali-metal surfaces we have used our EAM model to calculate the vibrational modes of oriented slabs of alkali-metal atoms. A more complete discussion of alkali-metal surface vibrations will be published elsewhere; here we illustrate our calculations with results for the $\mathrm{Na}(110)$ surface. We choose to discuss this specific surface because it has received the most theoretical and experimental attention of all the alkali-metal surfaces. In particular, we note that there are two prior calculations of $\mathrm{Na}(110)$ surface phonons, a preliminary one based on DFT by Rodach et al. [42] and a rather extensive EAM investigation by SBC [38, 39]. These calculations, however, did not consider near-surface Debye temperatures.

Because the allowed vibrational modes are modified by the finite thickness of the slab, the vibrational structure in the interior of the slab is not identical to that calculated for the bulk. The biggest difference is associated with long-wavelength modes that have wavelengths comparable to or longer than the thickness of the slab. Coupled with this effect is a dependence of the layer resolved Debye temperatures on the number of sampled $k$-space points. In order to determine the most reliable values for calculated 
Table 3. Surface relaxations of $\mathrm{Li}$ and $\mathrm{Na}$ low-index surfaces. Negative (positive) values signify inward (outward) relaxation. Values are the percentage of the interlayer spacing for a given surface. $\Delta_{i j}$ represents the change in distance (compared to the bulk) between layers $i$ and $j$.

\begin{tabular}{|c|c|c|c|}
\hline Surface & $\Delta_{12}(\%)$ & $\Delta_{23}(\%)$ & Technique \\
\hline $\operatorname{Li}(110)$ & $\begin{array}{r}-0.5 \\
-1.9 \\
-2.1 \\
1.3\end{array}$ & $\begin{array}{c}-0.06 \\
-0.08 \\
0.0\end{array}$ & $\begin{array}{l}\text { DFT b } \\
\text { EAM (present calculation) } \\
\text { EAM (GA) }{ }^{\text {e }} \\
\text { EAM }(\text { SBC })^{f}\end{array}$ \\
\hline $\mathrm{Na}(110)$ & $\begin{array}{l}0 \\
0 \\
-1.6 \pm 0.5 \\
-1.6 \\
-1.5 \\
2.4\end{array}$ & $\begin{aligned} & 0.0 \pm 0.5 \\
- & 0.0 \\
- & 0.07 \\
& 0.1\end{aligned}$ & $\begin{array}{l}\text { DFT }^{\text {a }} \\
\text { DFT }^{\text {b }} \\
\text { DFT }^{c} \\
\text { EAM (present calculation) } \\
\text { EAM (GA) }{ }^{\text {e }} \\
\text { EAM }(\text { SBC })^{\text {f }}\end{array}$ \\
\hline $\operatorname{Li}(100)$ & $\begin{array}{r}-3.0 \\
-3.2 \\
-2.6 \\
5.3\end{array}$ & $\begin{array}{c}-0.8 \\
-0.88 \\
0\end{array}$ & $\begin{array}{l}\text { DFT b } \\
\text { EAM (present calculation) } \\
\text { EAM (GA) }{ }^{\text {e }} \\
\text { EAM }(\text { SBC })^{f}\end{array}$ \\
\hline $\mathrm{Na}(100)$ & $\begin{array}{c}-2.0 \\
-0.7 \\
0 \\
-0.36 \\
-0.34 \\
8.6\end{array}$ & $\begin{array}{c}-1.1 \\
-0.91 \\
0.7\end{array}$ & $\begin{array}{l}\operatorname{DFT}^{\text {a }} \\
\operatorname{DFT}^{\text {b }} \\
\text { DFT }^{\text {d }} \\
\text { EAM (present calculation) } \\
\text { EAM (GA) e } \\
\text { EAM (SBC) }\end{array}$ \\
\hline
\end{tabular}

a $[40]$.
$\mathrm{b}[41]$.
$\mathrm{c}[42]$.
$\mathrm{d}[43]$.
$\mathrm{e}[12]$.
$\mathrm{f}[38,39]$.

Debye temperatures we have thus investigated their dependence on these two factors. We find for 1600 sampled $k$-space points that a slab thickness 30 times the lattice constant is sufficient to produce $n \geq-2$ center-layer Debye temperatures that are within $5 \%$ of bulk Debye temperatures, which we set as our criterion for accuracy. We note that because they are extremely sensitive to the longest wavelength modes, $n=-3$ Debye temperatures cannot be accurately calculated in a slab geometry with a reasonable number of layers and sampled $k$-space points.

Dispersion curves for $\mathrm{Na}(110)$ along high-symmetry directions in the surface Brillouin zone (SBZ) are shown in figure 3 for a 51 layer slab. The three panels of this figure highlight strong longitudinal (L), shear horizontal (SH), and shear vertical (SV) motions in the first layer with the degree of localization indicated by the degree 
of the shading. Strongly localized modes of all three symmetry types are found at this surface.

Although there is general overall qualitative agreement with vibrational spectra previously calculated by SBC using their EAM model [38, 39], the results shown in figure 3 have several notable differences. (i) The range of frequencies for a given wavevector are much smaller for our model than for the SBC model. For example, at $\bar{N}$ our calculated frequencies range from 0.8 to $3.8 \mathrm{THz}$ while those of the $\mathrm{SBC}$ model range from 0.3 to $4.5 \mathrm{THz}$. These differences are a consequence of the SBC model underestimating and overestimating the lowest and highest frequencies, respectively, along the bulk [110] direction [39]. (ii) The strong $\mathrm{SH}$ mode in the $\bar{\Gamma}$ to $\bar{H}$ direction was not identified by SBC. (iii) In the $\bar{\Gamma}$ to $\bar{N}$ direction the Rayleigh mode in the SBC calculation is significantly below the bottom of the bulk bands. For example, at $\bar{N} \mathrm{SBC}$ calculate the Rayleigh-mode frequency to be about half that of the lowest bulk mode. In contrast, along $\bar{\Gamma}$ to $\bar{N}$ our calculation predicts the Rayleigh mode to be just below the bulk bands. Observations (ii) and (iii) are likely related to the unphysical outward expansion of the first layer in the SBC model (see table 3).

Our calculated vibrational structure also has significant differences compared with results from the early DFT calculations of Rodach et al. [42]. The main differences in the spectra of Rodach et al. are (i) the absence of a first-layer longitudinal mode between $\bar{P}$ and $\bar{H}$ below the highest part of the bulk spectrum and (ii) the presence of a surface-localized mode above much of the bulk spectrum. These differences can be traced to a significant stiffening (compared to the bulk) of the surface force constants in the Rodach et al. model.

Further insight into near-surface vibrational dynamics is provided by layer resolved Debye temperatures, which are listed in table 4 for $n=-2,0$, and 2. Also listed are results for the center layer and results obtained from the bulk calculation. In addition to being layer resolved, the tabulated Debye temperatures are resolved for motions parallel ( $x$ and $y$ ) and perpendicular $(z)$ to the surface normal. Because the $n=-2$ Debye temperatures are most sensitive to longer wavelength modes, the center-layer values do not converge as readily to the bulk value as do the $n=0$ and 2 values, although all are within $5 \%$ of the bulk values.

The major qualitative aspects of the near-surface Debye temperatures can be understood by considering the coordination of the atoms in each layer. As table 4 shows, the Debye temperatures of the first atomic layer are all less than the corresponding Debye temperatures of deeper layers, and motion perpendicular to the surface is softer than parallel motion. The relative overall softness of the vibrations in the first layer is not unexpected as a first-layer atom is missing 2 of 8 nearest neighbor (NN) atoms and 2 of 6 next-nearest neighbor (NNN) atoms. The first-layer directional dependence can also be understood in terms of coordination as a first-layer atom has the same intralayer NN and NNN coordinations as a bulk atom, but is missing half of its out-of-layer nearest and next-nearest neighbors. For the bcc(110) surface a second-layer atom is coordinated by all of its nearest and next-nearest neighbors. Thus, on the length scale of $\sim 2 a_{0}$ the 

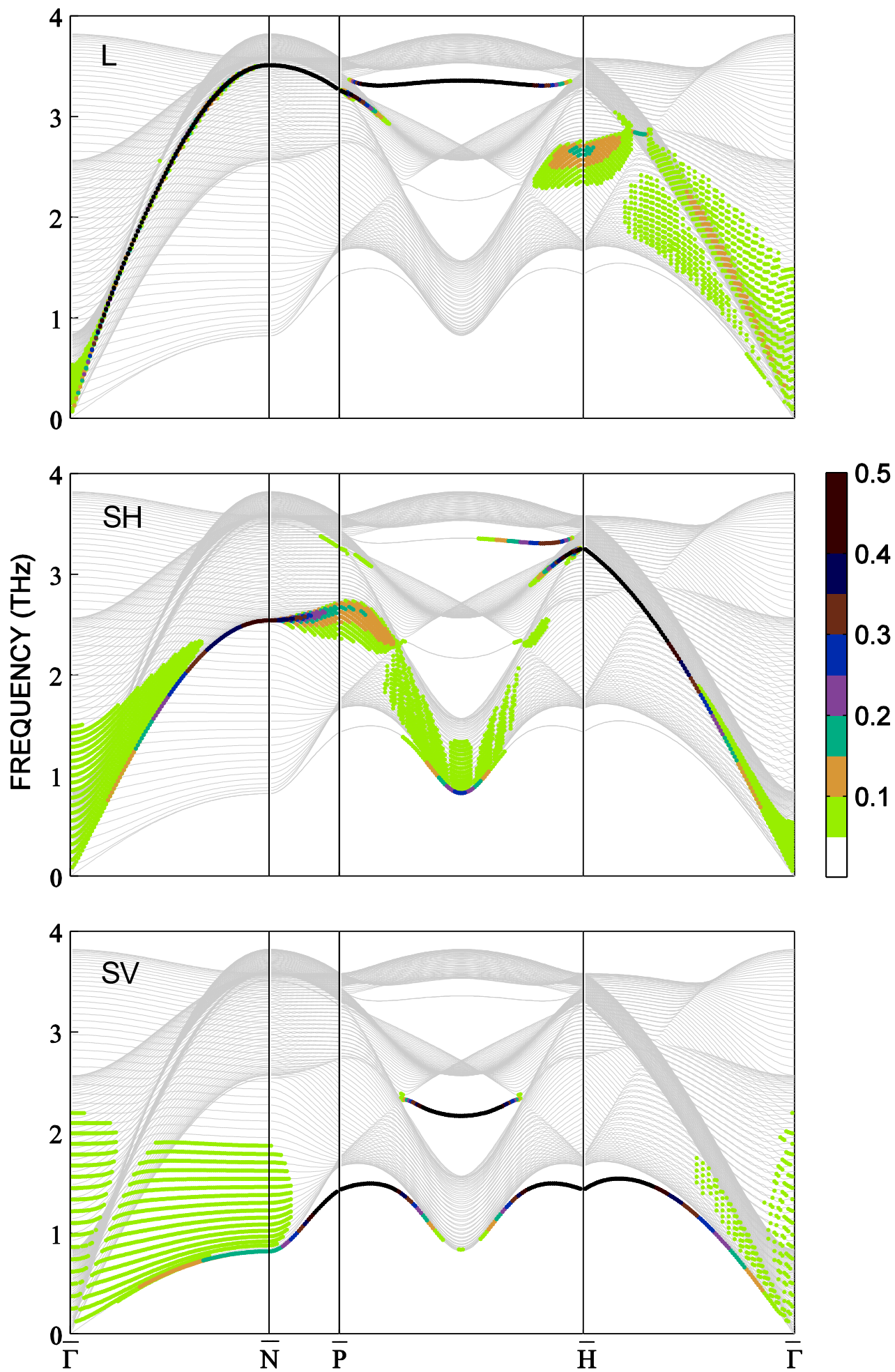

Figure 3. Phonon dispersion curves along high-symmetry directions of SBZ for a 51layer $\mathrm{Na}(110)$ slab. Strong longitudinal (L), shear horizontal (SH), and shear vertical (SV) motions in the first layer are highlighted, with the degree of shading indicating the degree of localization. 
Table 4. Directionally resolved frequency-moment Debye temperatures for layers parallel to the $\mathrm{Na}(110)$ surface for a 41-layer slab. The $x, y$, and $z$ directions are parallel to (110), (001), and (110) directions, respectively. Bulk Debye temperatures are also shown for comparison.

\begin{tabular}{|c|c|c|c|c|}
\hline Layer & $n$ & $x$ & $y$ & $z$ \\
\hline \multirow[t]{3}{*}{1} & -2 & 126 & 117 & 106 \\
\hline & 0 & 147 & 131 & 112 \\
\hline & 2 & 158 & 142 & 125 \\
\hline \multirow[t]{3}{*}{2} & -2 & 138 & 130 & 121 \\
\hline & 0 & 156 & 154 & 152 \\
\hline & 2 & 166 & 167 & 168 \\
\hline \multirow[t]{3}{*}{3} & -2 & 141 & 136 & 130 \\
\hline & 0 & 156 & 156 & 155 \\
\hline & 2 & 166 & 166 & 165 \\
\hline \multirow[t]{3}{*}{4} & -2 & 142 & 139 & 134 \\
\hline & 0 & 156 & 156 & 156 \\
\hline & 2 & 166 & 166 & 166 \\
\hline \multirow[t]{3}{*}{ center } & -2 & 145 & 144 & 142 \\
\hline & 0 & 157 & 157 & 157 \\
\hline & 2 & 166 & 166 & 166 \\
\hline \multirow[t]{3}{*}{ bulk } & -2 & 150 & & \\
\hline & 0 & 159 & & \\
\hline & 2 & 167 & & \\
\hline
\end{tabular}

environment of a second-layer atom is very bulk like, and so the higher order $(n=$ 0 and 2) Debye temperatures (which are more sensitive to shorter wavelength modes) are very close to the bulk Debye temperatures, while the $n=-2$ Debye temperatures (which are more sensitive to longer wavelength modes) are still significantly lower than the corresponding bulk Debye temperatures. As table 4 shows, even the fourth-layer, $n=-2$ Debye temperatures are somewhat lower than the center-layer values.

Quantitatively, our results are in good agreement with low energy electron diffraction (LEED) measurements by Andersson et al. of a Debye temperature at the $\mathrm{Na}(110)$ surface [44]. In that study the intensity of the specularly reflected beam was measured as a function of sample temperature between 180 and $270 \mathrm{~K}$. As (i) these measurements are at temperatures above $\sim 1 / 3$ of a typical Na Debye temperature, (ii) the specular beam corresponds to momentum transfer perpendicular to the surface, and (iii) the inelastic mean-free path of the incident electrons in the experiment is $\sim 0.5 \mathrm{~nm}[45]$, the measured Debye temperature corresponds to a near-surface averaged, $z$-direction, $n=-2$ Debye temperature. From their measurements Anderson et al. extracted Debye temperatures of 103, 114, and $113 \mathrm{~K}$ for incident beam energies 
of $14,35.5$ and $65.5 \mathrm{eV}$, respectively. These values are all very close to the average value $(113.5 \mathrm{~K})$ of our calculated first and second layer $n=-2$, $z$-direction Debye temperatures.

\section{Summary}

We have presented results for alkali-metal vibrational dynamics calculated with an analytic EAM model. Keys to the construction of the EAM potentials include (i) the use of the best available physical parameters (elastic constants, vibrational frequencies, etc.), (ii) a careful search for the best value of the pair-potential free parameter $\alpha$, and (iii) consideration of surface relaxation in setting the value of the embedding-energy exponent $\lambda$. As we have demonstrated, this model is able to accurately model bulk dispersion curves, frequency-moment Debye temperatures, and (temperature dependent) entropy Debye temperatures of all five alkali metals.

Given the model's accurate descriptions of bulk-phonon spectra and surface relaxation, we expect it to be able to accurately describe surface vibrations. We have illustrated this ability with calculations of vibrations at the $\mathrm{Na}(110)$ surface. The surface-phonon dispersion curves show significant differences when compared with other calculations of this surface. Satisfyingly, the near-surface $n=-2$, perpendicular Debye temperature is in excellent agreement with experimental LEED results. By investigating layer dependent directional Debye temperatures, we have gained insight into the nearsurface dynamics. Such near-surface information is important in the interpretation of a number of surface-sensitive techniques, including LEED, core-level photoemission, and grazing-incidence x-ray measurements. We are currently extending these calculations to other surfaces $[\mathrm{Na}(100), \mathrm{Na}(111)$, and $\mathrm{Na}(211)]$ to investigate the effects that surface orientation has on the near-surface vibrational dynamics.

\section{Appendix A. Lattice Stability}

Owing to the history of lattice-stability calculations with various EAM formulations, we include here a brief discussion of the relative stability of the bcc, fcc, and hcp lattices for the five alkali metals. Using our potentials we have calculated the cohesive energy for each lattice type; the cohesive energy differences $E_{f c c}-E_{b c c}$ and $E_{h c p}-E_{b c c}$ are reported in table A1. As indicated in the table, for all five alkali metals our potentials predict the bcc lattice to be more stable than either the fcc or hcp lattices, with the cohesive energy differences ranging from +0 to $9 \mathrm{meV}$. Insofar as our $E_{f c c}-E_{b c c}$ values for $\mathrm{Li}, \mathrm{Na}$, and $\mathrm{K}$ all lie within the respective range of EAM-calculated values that have been previously reported (also shown in table A1), our results are typical for an EAM model.

It is informative to compare the EAM calculated results with those of first-principles theory (FPT), which are also tabulated in table A1. Due to the transformation of Li from the bcc phase to a close-packed configuration as the temperature is decreased 
Table A1. Theoretical cohesive energy differences $E_{f c c}-E_{b c c}$ and $E_{h c p}-E_{b c c}$ for the alkali metals. A positive (negative) number indicates that bcc is the more (less) stable lattice. All values have been rounded to the nearest meV. Acronyms associated with the FPT results are from the individual references and serve to identify important aspects of the calculations; see references for details.

\begin{tabular}{|c|c|c|c|c|c|c|c|c|c|c|}
\hline \multicolumn{5}{|c|}{$E_{f c c}-E_{b c c}(\mathrm{meV})$} & \multicolumn{5}{|c|}{$E_{h c p}-E_{b c c}(\mathrm{meV})$} & \multirow{2}{*}{ Technique } \\
\hline $\mathrm{Li}$ & $\mathrm{Na}$ & $\mathrm{K}$ & $\mathrm{Rb}$ & Cs & $\mathrm{Li}$ & $\mathrm{Na}$ & $\mathrm{K}$ & $\mathrm{Rb}$ & Cs & \\
\hline 4 & 9 & 5 & 0 & 2 & 3 & 6 & 6 & 1 & 2 & EAM (this study) \\
\hline 21 & 36 & 28 & & & & & & & & EAM (JO) [10] \\
\hline 10 & 10 & 10 & & & -1 & -0 & -0 & & & EAM $[46]$ \\
\hline-11 & -0 & 1 & 1 & & & & & & & EAM (CM) [8] \\
\hline-1 & 0 & 1 & 1 & 1 & -0 & 0 & 1 & 1 & 1 & $\operatorname{EAM}(\mathrm{HM})[33]^{\mathrm{a}}$ \\
\hline 10 & & & & & 22 & & & & & EAM $[47]$ \\
\hline-12 & & & & & -12 & & & & & EAM [48] \\
\hline-2 & 0 & 0 & 0 & 0 & -2 & 0 & 0 & 0 & 0 & FPT (LMTO) [49] \\
\hline-6 & & & & & & & & & & $\mathrm{FPT}(\mathrm{KSG})[50]$ \\
\hline-12 & & & & & & & & & & $\mathrm{FPT}(\mathrm{RSK})[50]$ \\
\hline-3 & 1 & 1 & & & -9 & -2 & 5 & & & $\mathrm{FPT}(\mathrm{PW})[51]$ \\
\hline-3 & & & & & -4 & & & & & FPT (LMTO) [52] \\
\hline & & 2 & & & & & 2 & & & FPT (Rel LMTO) [53] \\
\hline-6 & -4 & & & & & & & & & FPT (FLAPW) [54] \\
\hline-3 & & & & & -4 & & & & & FPT (FLAPW) [55] \\
\hline-4 & & -1 & & & & & & & & FPT (FLAPW) [56] \\
\hline-4 & & & & & & & & & & FPT (PWGGA) [57] \\
\hline-7 & & & & & -8 & & & & & $\mathrm{FPT}(\mathrm{CC})[58]$ \\
\hline & & 1 & 1 & 0 & & & & & & $\mathrm{FPT}(\mathrm{LPM})[59]$ \\
\hline-3 & & -0 & -2 & & & & & & & FPT (FLAPW) [60] \\
\hline-2 & & & & & 0 & & & & & $\mathrm{FPT}(\mathrm{VWN})[61]$ \\
\hline-2 & & & & & -2 & & & & & $\mathrm{FPT}(\mathrm{PW})[62]$ \\
\hline-1 & & & & & -1 & & & & & FPT (PWGGA $4 s 3 p 1 d$ ) [63] \\
\hline
\end{tabular}

a Values are obtained from figure 5 of [33]; these not the same energy differences reported in table 3 of [33], which do not correspond to equilibrium fcc and hcp lattice constants.

below $80 \mathrm{~K}$ [37], the relative stability of different phases of Li has garnered the most attention. Taken en mass, the results of the first-principles calculations for Li indicate that the close-packed fcc and hcp phases are more stable than the bcc phase. For both $E_{f c c}-E_{b c c}$ and $E_{h c p}-E_{b c c}$ the average of the FPT calculations is $-4 \mathrm{meV}$ with a standard deviation of $3 \mathrm{meV}$. It has been previously noted that a result in this range is consistent with the experimentally observed transition temperature [54]. As shown in table A1, several EAM potentials do produce a negative value for $E_{f c c}-E_{b c c}$ and/or $E_{h c p}-E_{b c c}$ for Li. Using the FPT results as a standard, the model of HM [33] is the most accurate of the EAM models in describing alkali-metal lattice stability. 


\section{References}

[1] Daw M S and Baskes M I 1983 Phys. Rev. Lett. 501285

[2] Daw M S and Baskes M I 1984 Phys. Rev. B 296443

[3] Daw M S, Foiles S M and Baskes M I 1993 Materials Science Reports 9251

[4] Nelson J S, Sowa E C and Daw M S 1988 Phys. Rev. Lett. 611977

[5] Nelson J S, Daw M S and Sowa E C 1989 Phys. Rev. B 401465

[6] Nelson J, Daw M and Sowa E C 1990 Superlattices and Microstructures 7259

[7] Benedek G, Bernasconi M, Chis V, Chulkov E, Echenique P M, Hellsing B and Toennies J P 2010 J. Phys.: Condens. Matter 22084020

[8] Chantasiriwan S and Milstein F 1998 Phys. Rev. B 585996

[9] Wang Y R and Boercker D B 1995 Journal of Applied Physics 78122

[10] Johnson R A and Oh D J 1989 Journal of Materials Research 41195

[11] Johnson R A 1988 Phys. Rev. B 373924

[12] Guellil A M and Adams J B 1992 Journal of Materials Research 7639

[13] Kittel C 2005 Introduction to Solid State Physics (New York: Wiley)

[14] MacDonald D K C 1953 The Journal of Chemical Physics 21177

[15] Adlhart W, Fritsch G and Lscher E 1975 Journal of Physics and Chemistry of Solids 361405

[16] Mundy J N, Miller T E and Porte R J 1971 Phys. Rev. B 3(8) 2445

[17] Martin D L 1965 Phys. Rev. 139 A150

[18] Anderson M S and Swenson C A 1985 Phys. Rev. B 31668

[19] Siegel S and Quimby S L 1938 Phys. Rev. 5476

[20] Barrett C S 1956 Acta Crystallographica 9671

[21] Schouten D R and Swenson C A 1974 Phys. Rev. B 102175

[22] Slotwinski T and Trivisonno J 1969 Journal of Physics and Chemistry of Solids 301276

[23] Quimby S L and Siegel S 1938 Phys. Rev. 54293

[24] Martinson R H 1969 Phys. Rev. 178902

[25] Marquardt W and Trivisonno J 1965 Journal of Physics and Chemistry of Solids 26273

[26] Gutman E and Trivisonno J 1967 Journal of Physics and Chemistry of Solids 28805

[27] Kollarits F and Trivisonno J 1968 Journal of Physics and Chemistry of Solids 292133

[28] Smith H G, Dolling G, Nicklow R M, Vijayaraghavan P R and Wilkinson M K 1968 Neutron Inelastic Scattering 1149

[29] Woods A D B, Brockhouse B N, March R H, Stewart A T and Bowers R 1962 Phys. Rev. 128 1112

[30] Cowley R A, Woods A D B and Dolling G 1966 Phys. Rev. 150487

[31] Copley J R D and Brockhouse B N 1973 Canadian Journal of Physics 51657

[32] Nücker N and Buchenau U 1985 Phys. Rev. B 315479

[33] Hu W and Masahiro F 2002 Modelling Simulation Mater. Sci. Eng. 10707

[34] Zhang J M, Zhang X J and Xu K W 2008 Journal of Low Temperature Physics 150730

[35] Xie Y and Zhang J M 2008 Canadian Journal of Physics 86801

[36] Grimvall G 1981 The Electron-Phonon Interaction in Metals (New York: Oxford)

[37] Schwarz W, Blaschko O and Gorgas I 1991 Phys. Rev. B 446785

[38] Sklyadneva I Y, Chulkov E V and Bertsch A V 1996 Surface Science 352-354 25

[39] Sklyadneva I Y, Berch A V and Chulkov E V 1995 Phys. Solid State 371454

[40] Bohnen K P 1982 Surface Science $\mathbf{1 1 5}$ L96

[41] Bohnen K P 1984 Surface Science 147304

[42] Rodach T, Bohnen K P and Ho K M 1989 Surface Science 209481

[43] Quong A A, Maradudin A A, Wallis R F, Gaspar J A, Eguiluz A G and Alldredge G P 1991 Phys. Rev. Lett. 66743

[44] Andersson S, Pendry J and Echenique P 1977 Surface Science 65539

[45] Wertheim G K, Riffe D M, Smith N V and Citrin P H 1992 Phys. Rev. B 461955 
[46] Baskes M I 1992 Phys. Rev. B 462727

[47] Yuan X, Takahashi K, Yin Y and Onzawa T 2003 Modelling Simulation Mater. Sci. Eng. 11447

[48] Cui Z, Gao F, Cui Z and Qu J 2012 Modelling Simulation Mater. Sci. Eng. 20015014

[49] Skriver H L 1985 Phys. Rev. B 31 1909-1923

[50] Boettger J C and Trickey S B 1985 Phys. Rev. B 323391

[51] Dacorogna M M and Cohen M L 1986 Phys. Rev. B 344996

[52] Boettger J C and Albers R C 1989 Phys. Rev. B 393010

[53] Alouani M, Christensen N E and Syassen K 1989 Phys. Rev B 398096

[54] Sigalas M, Bacalis N C, Papaconstantopoulos D A, Mehl M J and Switendick A C 1990 Phys. Rev. B 4211637

[55] Nobel J A, Trickey S B, Blaha P and Schwarz K 1992 Phys. Rev. B 455012

[56] Papaconstantopoulos D A and Singh D J 1992 Phys. Rev. B 457507

[57] Perdew J P, Chevary J A, Vosko S H, Jackson K A, Pederson M R, Singh D J and Fiolhais C 1992 Phys. Rev. B 466671

[58] Cho J H, Ihm S H and Kang M H 1993 Phys. Rev. B 4714020

[59] Mutlu R H 1995 Phys. Rev. B 521441

[60] Sliwko V L, Mohn P, Schwarz K and Blaha P 1996 J. Phys.: Condens. Matter 8799

[61] Staikov P, Kara A and Rahman T S 1997 J. Phys.: Condens. Matter 92135

[62] Liu A Y, Quong A A, Freericks J K, Nicol E J and Jones E C 1999 Phys. Rev. B 594028

[63] Doll K, Harrison N M and Saunders V R 1999 Journal of Physics: Condensed Matter 115007 